\title{
Mobile science
}

\section{Smartphones that have been cleverly 'accessorized' are starting to offer a convenient and cost-effective alternative to conventional laboratory-based imaging and sensing equipment.}

Smartphones have entered our lives in recent years; and arguably changed it entirely. There are nearly 7 billion mobile phone subscriptions worldwide ${ }^{1}$ - equivalent to $95.5 \%$ of the world's population and almost 1.5 billion of them are for smartphones ${ }^{2}$. We now photograph anything, at any time, and upload pictures to social networks, video-call our loved ones and routinely search the internet and send e-mails using our mobile phones.

Having already revolutionized the way we organize our social lives, it seems that the new trend is to use the advanced image sensor and computing power of customized phones for performing sophisticated healthcare tests and sensing tasks.

"Mobile phones and other consumer electronics devices have been emerging as powerful platforms to create costeffective, portable and readily accessible alternatives to some of the advanced biomedical imaging and measurement tools," explained Aydogan Ozcan, a professor the University of California, Los Angeles, who is actively exploring opportunities for using smartphones in photonics and healthcare applications. "Mobile phones have been experiencing massive advances in their optical imaging hardware, approximately doubling their space-bandwidth product every two years over the past $\sim 10-15$ years, recently reaching to more than 40 million pixels in their digital camera systems."

Several research groups and companies worldwide are starting to exploit the image sensor within phones for a variety of tasks, with ophthalmology being the most popular application to date. An MIT team has developed a cheap phonebased prototype device called NETRA for conducting optometry ${ }^{3}$. The US healthcare company Welch Allyn has already launched its iExaminer accessory ${ }^{4}$, which interfaces an iPhone with an ophthalmoscope (pictured) and the UK start-up Peek Vision ${ }^{5}$ is conducting trials of a phone-based eye testing system.

Other basic microscopy tasks have also become a reality, including biomedical sensing of analytes in bodily fluids ${ }^{6}$, monitoring of food (bacteria, allergens and hormones) $)^{7}$ as well as environmental monitoring. And on page 784 of this issue,

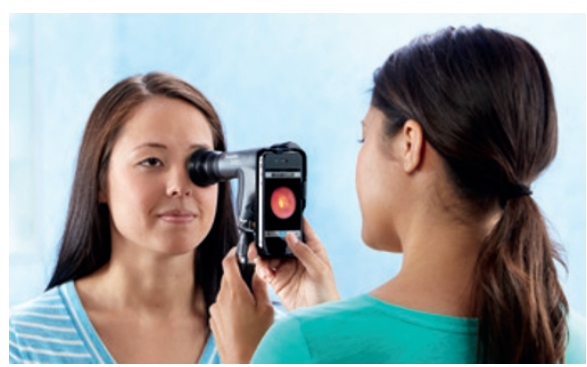

The Welch Allyn iExaminer makes it possible for an iPhone to record, store, send and retrieve images from an ophthalmoscope. The product became commercially available in the USA in January 2012.

Ori Katz and co-workers report a means of imaging through a scattering material implemented using a Nokia mobile phone.

Digital microscopy is only the start, however. Biomedical optical sensing that exploits the data connectivity of mobile phones also shows much promise for telemedicine and mobile medical applications. In this direction, several significant achievements have been recently reported. It is now possible to detect single viruses and nanoparticles using mobilephone-based fluorescent microscopes ${ }^{8}$. The Erickson Lab at Cornell University has developed a smartphone accessory for accurately measuring cholesterol levels within a minute?

A multidisciplinary team - which includes the group of Professor Erickson - has been awarded a US $\$ 3$ million grant for the so-called PHeNoM (Public Health, Nanotechnology and Mobility) program ${ }^{10}$. The aim is to develop smartphone-based platforms for long-term stress management (Stress-phone), nutritional awareness (Nutri-Phone) and for monitoring viral loading in HIV positive patients (Hema-phone).

The examples described above clearly indicate the prospects of smartphones within the healthcare sector. One can easily envision a future where standard medical tests currently performed in labs will be performed at home: blood analysis, estimating cholesterol levels, diagnosis of infectious diseases, monitoring chronic allergies, electrochemical detection of parasites, monitoring of electrocardiogram rhythms, detection of cataracts and so much more. Measurements are made with the press of a button and another tap sends the $\succeq$ results to the family doctor.

However, there are certain bumps on the road to getting there. The most obvious problem is the one of standardization across different phone brands and platforms. The use of open software and hardware platforms for mobile phones could be a solution, says Professor Ozcan, citing Android OS and the recently announced Project Ara ${ }^{11}$ (a Google initiative for a free, Lego-like open hardware platform for creating highly modular smartphones). Professor Erickson is equally optimistic. The reality is that in countries like the USA, handset variability is not a real issue; the vast majority of smartphones sold operate on either Apple's iOS or Google's Android platform ${ }^{12}$. The problem becomes more obvious in developing countries however, where tens of lower-cost brands of smartphones running other operating systems are available.

There is also another important aspect to creating cost-effective and yet powerful micro- and nano-imaging interfaces for mobile phones. It revolutionizes the possibilities for conducting research in the developing world. Cheaper mobile phone cameras are a powerful alternative to prohibitively expensive laboratory equipment and could be a useful alternative for researchers as well as students in schools and universities. According to Professor Ozcan, smartphones can potentially offer the possibility to overcome budget cuts and help support the "democratization of measurement science and higher education".

In summary, the future looks bright don't forget to charge your phones.

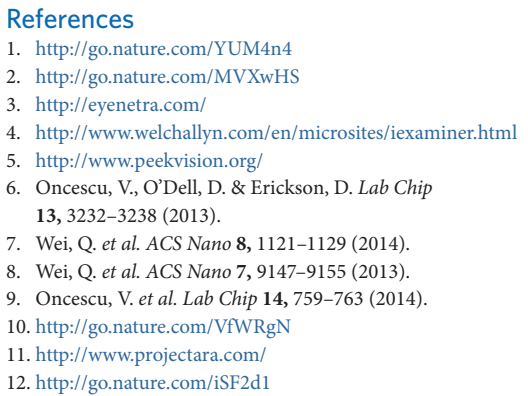

